\title{
Short-arc tracklet association for geostationary objects
}

\author{
J.A. Siminski*, O. Montenbruck, H. Fiedler \\ German Space Operations Center, Deutsches Zentrum für Luft- und Raumfahrt, 82234 Weßling, Germany
}

T. Schildknecht

Astronomical Institute, University of Bern, Sidlerstr. 5, CH-3012 Bern, Switzerland

\begin{abstract}
Space debris populating the geostationary orbit is a hazardous threat to active satellites and motivates a routine surveillance of this orbital region by ground-based optical telescopes. Due to limited resources short measurement arcs, called tracklets, are collected that do not provide sufficient information to determine full orbital states of the measured objects. The paper proposes a method to determine the orbit of an object using the available information of two tracklets, i.e. their line-of-sights and their derivatives. The line-of-sights at both observation epochs are augmented with range hypotheses in order to obtain possible orbit candidates. The derivatives of the line-of-sights are used to determine whether an orbit hypothesis fits to the actual measured tracklets or not. Computational optimization schemes are exploited to find the best hypotheses. If a hypothesis is found that approximates both tracklets sufficiently, they are both associated to each other. The association and run-time performance is assessed using real measurements.
\end{abstract}

Keywords: space situational awareness, initial orbit determination, tracklet association, too-short-arcs, admissible region

\section{Introduction}

In order to avoid proximities of active satellites and uncontrolled space objects or eventually to remove the debris, space object catalogs must be maintained. The geostationary orbit has unique characteristics in many respects. The orbit is of special importance as it is intensively used for communication, navigation and weather monitoring. Additionally, it is special because of its far distance from the surface of the Earth. Due to the limited range capabilities of radar antennas it is usually observed with optical telescopes. Considering a limited amount of telescopes

\footnotetext{
* Corresponding author

Email addresses: jan.siminski@dlr.de (J.A. Siminski), oliver.montenbruck@dlr.de (O. Montenbruck),

hauke.fiedler@dlr.de (H. Fiedler),

thomas.schildknecht@aiub.unibe.ch (T. Schildknecht)
}

Preprint submitted to Advances in Space Research that try to cover the complete orbital region, each object can only be tracked for a limited duration. The resulting short observation arcs, called tracklets, lack of complete state information and are therefore either associated to already cataloged objects (cf. with the procedure described in Früh and Schildknecht (2009)) or tested pairwise with other uncorrelated observations. The latter problem is approached in this work, i.e. two tracklets are examined whether they originate from the same object or not and, if they do, the common orbit solution is determined. This measurement association is a fundamental task during the catalog build-up phase for the initial location of space objects but also later for the relocation of lost ones. 


\subsection{Observations}

Each measurement arc contains a series of right ascension and declination values as measured by a topocentric observer. The information of the series, which is exploited for the association, is the line-of-sight $\mathbf{u}$ and its derivative $\dot{\mathbf{u}}$. It can also be represented by the angles and angular rates, commonly known as the attributable vector (c.f. (Milani et al., 2004))

$$
\mathbf{a}=(\alpha, \dot{\alpha}, \delta, \dot{\delta})^{\top}
$$

\subsection{Problem formulation}

The orbital motion of an object is described by six first order ordinary differential equations, where the vector form of the system is shown below

$$
\dot{\mathbf{y}}(t)=\mathbf{f}(t, \mathbf{y}(t)) \quad \text { where } \quad \mathbf{y}(t)=\left(\begin{array}{c}
\mathbf{r}(t) \\
\dot{\mathbf{r}}(t)
\end{array}\right) .
$$

The state of the system is uniquely defined by six constraints, i.e. an orbit solution is either defined by an initial value

$$
\mathbf{y}\left(t_{1}\right)=\left(\begin{array}{c}
\mathbf{r}_{1} \\
\dot{\mathbf{r}}_{1}
\end{array}\right)
$$

or by two boundary values

$$
\mathbf{y}_{1: 3}\left(t_{1}\right)=\mathbf{r}_{1} \quad \text { and } \quad \mathbf{y}_{1: 3}\left(t_{2}\right)=\mathbf{r}_{2},
$$

where in the first case the position and velocity at one epoch must be provided and in the latter case the positions at two observation epochs. The elements of the state vector can be described in terms of the observed variables. The geocentric position

$$
\mathbf{r}(\rho)=\mathbf{r}_{s}+\rho \mathbf{u}
$$

is dependent on the line-of-sight information, the sensor position, and the unobserved range $\rho$. The velocity

$$
\dot{\mathbf{r}}(\rho, \dot{\rho})=\dot{\mathbf{r}}_{s}+\rho \dot{\mathbf{u}}+\dot{\rho} \mathbf{u}
$$

additionally requires knowledge on the line-of-sight derivative, the sensor velocity, and the unobserved range-rate $\dot{\rho}$.
Therefore, one tracklet constrains the state of an object in four degrees of freedom with two unknown parameters $(\rho, \dot{\rho})$. Thus, to uniquely determine a state of an object, at least two observation arcs are required. Hence, an overdetermined system with eight known and four free parameters, namely the range and range-rate at both observation epochs, is obtained. The tracklet association problem is illustrated in Fig. 1.

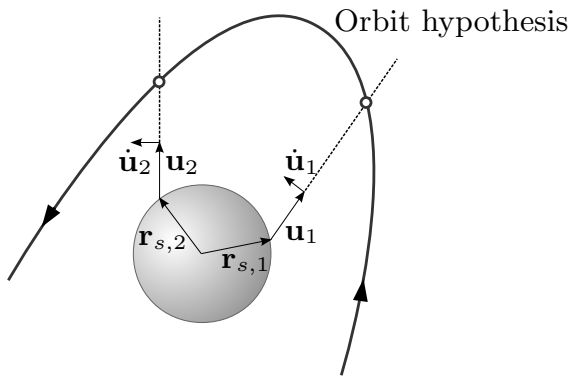

Figure 1: Illustration of the two tracklet association problem: Shown are two line-of-sight vectors $\mathbf{u}_{1}$ and $\mathbf{u}_{2}$ and their derivatives at different epochs and with different station coordinates $\mathbf{r}_{s, 1}$ and $\mathbf{r}_{s, 2}$. Furthermore an orbit hypothesis connecting both observations is shown.

To date various methods have been developed to approach the tracklet association problem. All methods hypothesize some of the unknown free parameters to obtain an orbit estimate. This hypothesis is then tested for its suitability. The methods differ, however, in the way the orbit is represented, i.e. using the initial value or the boundary value formulation. Table 1 gives an overview of current approaches and their relation to each other. The different formulations and methods are recapitulated in the following.

\section{Initial-value method}

When augmenting the tracklet information from the first observation epoch with the two free parameters $\left(\rho_{1}, \dot{\rho}_{1}\right)$, a full state is defined

$$
\hat{\mathbf{y}}\left(t_{1}\right)=\left(\begin{array}{c}
\mathbf{r}\left(\rho_{1}\right) \\
\dot{\mathbf{r}}\left(\rho_{1}, \dot{\rho}_{1}\right)
\end{array}\right)
$$




\begin{tabular}{|c|c|c|c|c|}
\hline Orbit representation & \multicolumn{2}{|l|}{ Parameters } & \multicolumn{2}{|l|}{ Approaches } \\
\hline \multirow{3}{*}{$\begin{array}{l}\text { Initial value } \\
\mathbf{r}_{1} \text { and } \dot{\mathbf{r}}_{1}\end{array}$} & \multirow{3}{*}{$\begin{array}{l}\text { State } \\
\text { Hypotheses } \\
\text { Discriminator }\end{array}$} & \multirow{3}{*}{$\begin{array}{l}\left(\alpha_{1}, \dot{\alpha}_{1}, \delta_{1}, \dot{\delta}_{1}\right) \\
\left(\rho_{1}, \dot{\rho}_{1}\right) \\
\left(\alpha_{2}, \dot{\alpha}_{2}, \delta_{2}, \dot{\delta}_{2}\right)\end{array}$} & Regular grid testing & DeMars and Jah (2013) \\
\hline & & & Hyperplane intersection & Fujimoto et al. (2013) \\
\hline & & & Optimization & Siminski et al. $(2013 a, b)$ \\
\hline \multirow{2}{*}{$\begin{array}{l}\text { Boundary value } \\
\qquad \mathbf{r}_{1} \text { and } \mathbf{r}_{2}\end{array}$} & \multirow{2}{*}{$\begin{array}{l}\text { State } \\
\text { Hypotheses } \\
\text { Discriminator }\end{array}$} & \multirow{2}{*}{$\begin{array}{l}\left(\alpha_{1}, \delta_{1}, \alpha_{2}, \delta_{2}\right) \\
\left(\rho_{1}, \rho_{2}\right) \\
\left(\dot{\alpha}_{1}, \dot{\delta}_{1}, \dot{\alpha}_{2}, \dot{\delta}_{2}\right)\end{array}$} & Regular grid testing & Schumacher et al. (2013) \\
\hline & & & Optimization & Presented approach \\
\hline
\end{tabular}

Table 1: Overview of the current tracklet association approaches.

which can be used as an initial value to numerically or analytically integrate the equation of motion (2) to the second epoch. Consequently, the solution $\hat{\mathbf{y}}\left(t_{2}\right)$ can be computed. The observation arc at the second epoch $t_{2}$ serves as a discriminator to decide whether a hypothesis is accepted or rejected.

Three different methods have been published which use this formulation. They all have in common that the solution space of the problem is beforehand limited to a so called admissible region. Milani et al. (2004) and Tommei et al. (2007) suggest to restrict the range and range-rate space by allowing only solutions that orbit the Earth on stable orbits, i.e. the energy of the orbit hypotheses must be negative and the range or perigee height must be above a certain limit. The latter assures that the candidate solution does not de-orbit within the next revolutions. If only a specific orbital region is of interest, the extend of the region can be furthermore reduced by specifying semi-major axis or eccentricity bounds.

\subsection{Regular grid testing}

DeMars and Jah (2013) sample the admissible region of the first tracklet with a bank of hypotheses on a regular grid. Each $\left(\rho_{1}, \dot{\rho}_{1}\right)$ - hypothesis is represented by a Gaussian, effectively a state $\hat{\mathbf{y}}\left(t_{1}\right)$ and covariance. This so called Gaussian mixture distribution is then updated given the following measurements. Hence, modeled measurements $\hat{\mathbf{a}}_{2}$ are computed from the propagated state hypotheses at the next epoch $\hat{\mathbf{y}}\left(t_{2}\right)$ and tested with the actual other ob- servation $\mathbf{a}_{2}$. If the association probability falls below a threshold, the component is removed from the distribution. The surviving hypotheses create candidate objects, which are affirmed and refined with further observations.

\subsection{Hyperplane intersection}

Fujimoto et al. (2013) find the common solution by computing the overlap of two reduced solutions spaces. Each admissible region is discretized with $\left(\rho_{1}, \dot{\rho}_{1}\right)$ - and $\left(\rho_{2}, \dot{\rho}_{2}\right)$ - hypotheses, which are represented by uniform probability density functions. All hypotheses are then propagated to a common epoch and compared to each other in some orbital elements space, which effectively is an intersection of two hyperplanes. The intersecting hypotheses are affirmed or rejected using a least-squares fit.

Both methods are illustrated in Fig. 2, where the dashed lines represent the process flow from Fujimoto et al. (2013) and the solid lines the one from DeMars and Jah (2013).

\subsection{Optimization}

In previous work a method was introduced that searches trough the free parameter space rather then evaluating all possible points on a regular grid (Siminski et al., 2013b). As in the approach by DeMars and Jah (2013) the hypothesis states are defined at one epoch only. However, the association probability is not evaluated for all points on the grid but computational optimization schemes are 


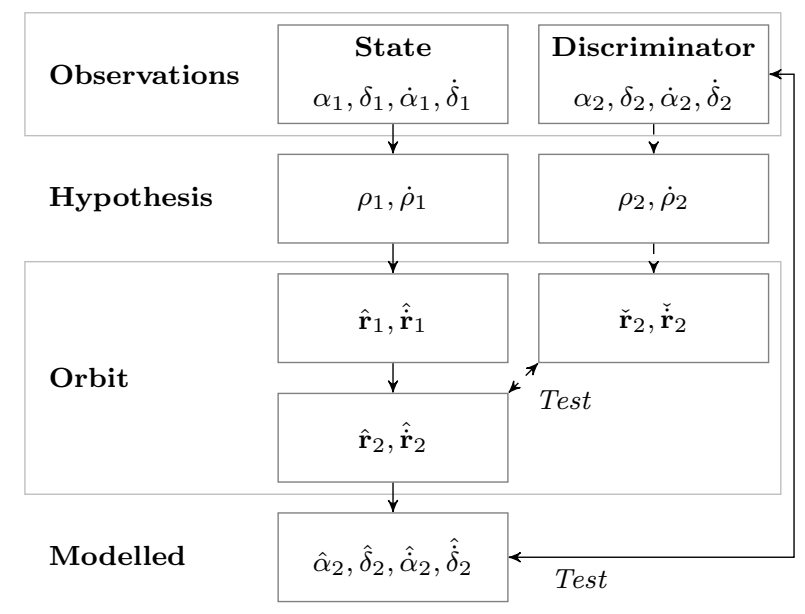

Figure 2: Process flow of the initial-value methods. The dashed path denotes the testing approach using hyperplane intersection.

exploited instead to identify wether two observations originate from the same object or not. Thus, a loss function is minimized which describes the difference between the actual second observation $\mathbf{a}_{2}$ and the predicted value $\hat{\mathbf{a}}_{2}$. As the measurements are affected by noise, the difference in the loss function is scaled with its uncertainty.

The topography of the loss function for the free parameters $\left(\rho_{1}, \dot{\rho}_{1}\right)$ is illustrated in Fig. 3, where the same geostationary object is re-observed after 3.75 days. The semi-major axis and orbital period stay constant on the dashed lines. The numbers in brackets denote the range of revolutions $k$ (in orbital periods) in the enclosed regions. The true solution is in the range $k \in[3.5,4]$. The topography of the loss function has been analyzed in (Siminski et al., 2013b) to assess the suitability for computational optimization methods. Several hills and valleys can be observed and, consequently, several local minima inside the valleys can be found that are potential candidates for a common orbit solution of the two measurement arcs. This results in a multi-modal optimization problem and makes it difficult to find a global minimum within the admissible region. When using iterative optimization methods, the location of the resulting minimum is largely dependent on the initial search value, e.g. it is likely to find the local

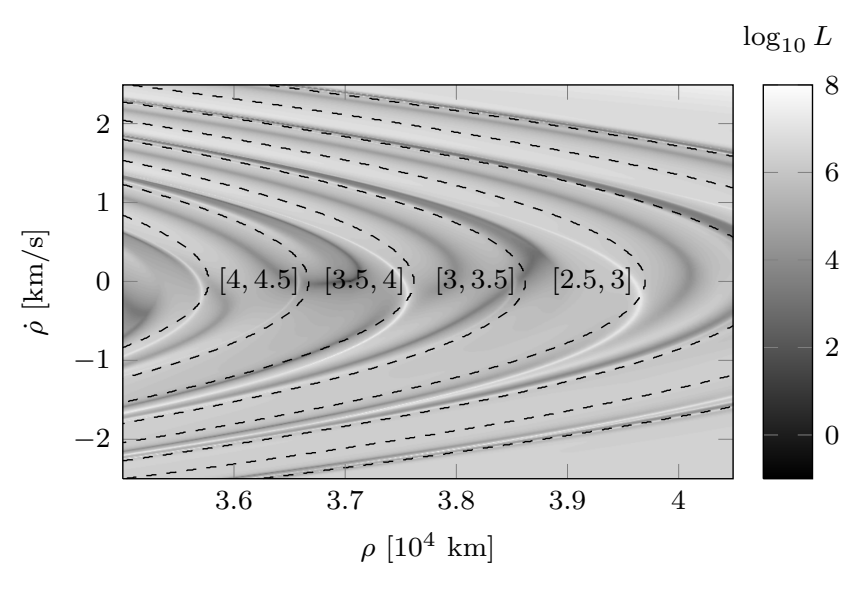

Figure 3: Loss function for initial-value method with range and range-rate as the free parameters. The same geostationary object at the longitude $\lambda=10^{\circ}$ has been re-observed after 3.75 days. The observing sensor is located at the same longitude and latitude $\phi=$ $45^{\circ}$.

minimum in the same valley in which the initial value is located.

For that reason, the individual valleys must be identified beforehand. They can be roughly separated by allowing only a specific range of orbital revolutions $k$ between the observation epochs. However, this can lead to very narrow regions as observed in Fig. 3, which is disadvantageous for iterative search algorithms as these might step outside the feasible area too easily. An appropriate alternative parameter system has been therefore proposed in (Siminski et al., 2013a) to account for the difficult topography. The semi-major axis $a_{1}$ and a relative range $\tilde{\rho}_{1}$ at the first epoch are used as the free parameters. The derivation and description of the new parameters can be obtained from the previously mentioned paper.

The topography of the loss function for the new set of parameters is illustrated in the following Figure 4. It shows a more suitable pattern for optimization algorithms. The representation allows to split the loss function into rectangles with the same number of orbital revolutions. Even though most valleys have been successfully separated from each other, multiple minima inside an orbital revolutions range can still be seen. Further research must find ways 


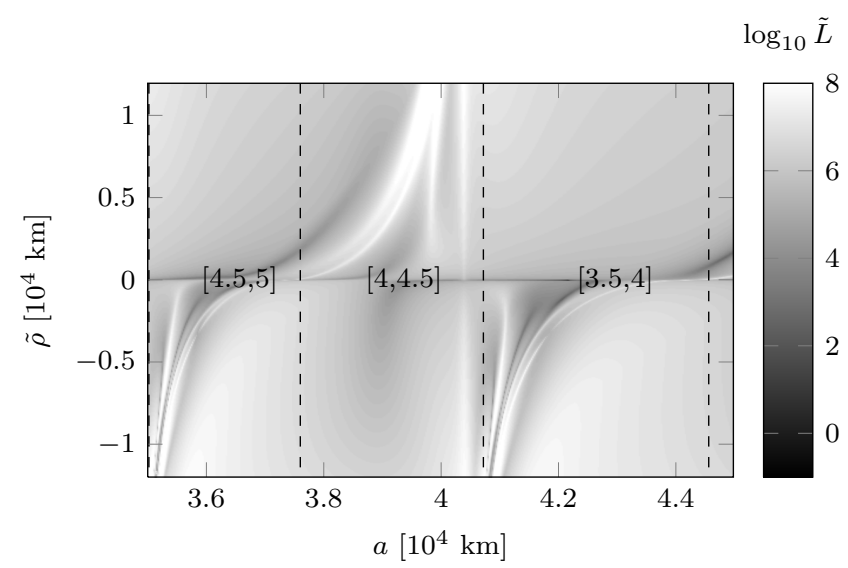

Figure 4: Loss function for initial-value method with semi-major axis and relative range as the free parameters. The same observations are used as in the previous Fig. 3.

to identify the different valleys inside such a range. For now, it is still required to solve a multi-modal optimization problem.

\section{Boundary-value method}

Instead of parametrizing the problem at the initial epoch only, more symmetric approaches have been developed that use the boundary-value formulation to define a state hypothesis. The line-of-sight information at both observation epochs

$$
\mathbf{z}=\left(\alpha_{1}, \delta_{1}, \alpha_{2}, \delta_{2}\right)^{\top}
$$

is used to constrain the state of the tested hypotheses. A complete orbital state is then defined by augmenting the line-of-sight information with unknown range values at both epochs $\left(\rho_{1}, \rho_{2}\right)$

$$
\hat{\mathbf{y}}_{1: 3}\left(t_{1}\right)=\mathbf{r}\left(\rho_{1}\right) \quad \text { and } \quad \hat{\mathbf{y}}_{1: 3}\left(t_{2}\right)=\mathbf{r}\left(\rho_{2}\right) .
$$

\subsection{Regular grid testing}

Schumacher et al. (2013) propose to use only the lineof-sight information if the angular rates are not available within a sufficient accuracy. Therefore, they sample a feasible range space for all measurements. Then, each range hypothesis of one measurement is combined with the range hypotheses of the other measurements. An orbit is determined for each $\left(\rho_{1}, \rho_{2}\right)$ - hypothesis using the solution to the Lambert's problem. The generated orbit hypotheses are affirmed and refined with further measurements.

Ansalone and Curti (2013) present a method for an observer in a low earth orbit, which uses the same idea. They sample the feasible range space on a coarse grid and test each hypothesis with the individually measured angles of the two tracklets. The fitness of each hypotheses is then iteratively improved using a genetic algorithm.

\subsection{Optimization}

This work introduces a method, where again an optimization scheme will be used to identify tracklets of common objects. The respective range values at both epochs are again chosen as free parameters to create candidate solutions. But, instead of testing the orbit hypotheses with further measurements or the individually measured angles, the angular rates

$$
\dot{\mathbf{z}}=\left(\dot{\alpha}_{1}, \dot{\delta}_{1}, \dot{\alpha}_{2}, \dot{\delta}_{2}\right)^{\top}
$$

are taken into account to decide whether a solution is suitable or not. These parameters are then used to define a loss function which is minimized to find the best fitting orbit to both observations.

Fig. 5 illustrates the process flow of the boundary-value methods. The dashed line represents the present approach, while the method proposed by Schumacher et al. (2013) uses additional measurements.

\section{Detailed derivation of the optimization method}

The two range hypotheses are combined for convenience in one hypothesis variable $\mathbf{p}=\left(\rho_{1}, \rho_{2}\right)$. When a $\mathbf{p}$ - hypothesis is given, the geocentric position vectors $\hat{\mathbf{r}}_{1}$ and $\hat{\mathbf{r}}_{2}$ are obtained using the line-of-sight information $\mathbf{z}$ and the sensor positions at the respective epochs. This forms 


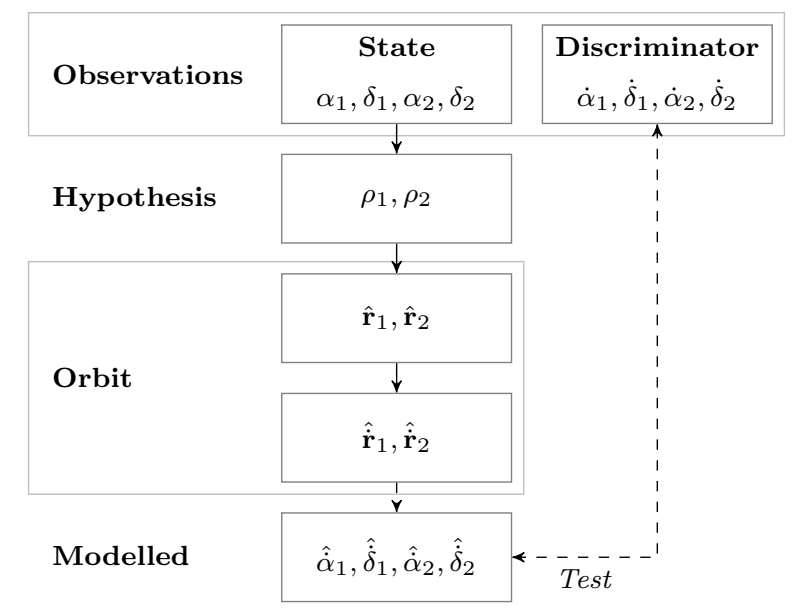

Figure 5: Process flow of the boundary-value methods. The dashed line denotes the new testing metric.

a special case of an orbital boundary value problem, commonly known as Lambert's problem, where the boundaries for the equation of motion (2) are constrained by two position vectors at epochs $t_{1}$ and $t_{2}$.

Several researchers solved it using geometrical considerations for non-perturbed two-body dynamics. Practical implementations can be obtained from e.g Gooding (1990) or Battin (1999). As the problem allows multiple solutions depending on the transfer path and the number of orbital periods, the number of full and half revolutions $k$ needs to be beforehand specified. If the fractional part of $k$ is larger than 0.5 , the long transfer path around the orbital ellipse is chosen. Otherwise, the solution is computed for the short way. The solution to the problem, effectively

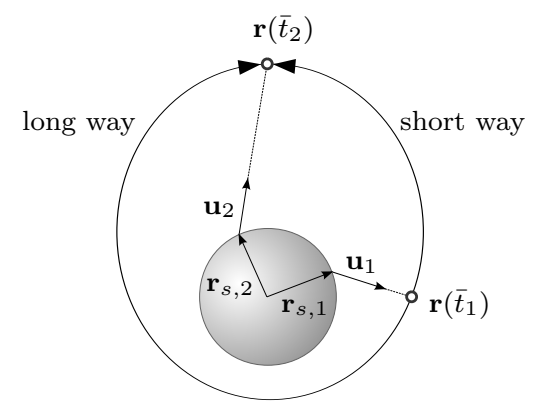

Figure 6: Illustration of Lambert's problem. The two possible transfer path directions are demonstrated. a set of orbital elements, is then used to determine the geocentric velocities $\hat{\dot{\mathbf{r}}}_{1}$ and $\hat{\dot{\mathbf{r}}}_{2}$ at both observation epochs. From these, the modeled topocentric rates

$$
\hat{\dot{\mathbf{z}}}(\mathbf{z}, \mathbf{p}, k)=\left(\hat{\dot{\alpha}}_{1}, \hat{\dot{\delta}}_{1}, \hat{\dot{\alpha}}_{2}, \hat{\dot{\delta}}_{2}\right)^{\top}
$$

are obtained.

As discussed before, the four remaining observables $\dot{\mathbf{z}}$ are utilized in a loss function as a discriminator

$$
L(\mathbf{p}, k)=(\dot{\mathbf{z}}-\hat{\mathbf{z}})^{\top}\left(\mathbf{C}_{\dot{\mathbf{z}}}+\mathbf{C}_{\hat{\dot{\mathbf{z}}}}\right)(\dot{\mathbf{z}}-\hat{\dot{\mathbf{z}}}),
$$

where not only the parameters $\mathbf{p}$ are unknown, but also the number of revolutions and the transfer path, i.e. the variable $k$. The covariance matrix $\mathbf{C}_{\dot{\mathbf{z}}}$ describes the uncertainties in the angular rates from (10) and $\mathbf{C}_{\hat{\mathbf{z}}}$ represents the effect of the angular uncertainties from (8) on the uncertainty of the modeled angular rates $\hat{\mathbf{z}}$.

The covariance matrix $\mathbf{C}_{\mathbf{z}}$ for the angles must be propagated using

$$
\mathbf{C}_{\hat{\mathbf{z}}}=\left(\frac{\partial \hat{\dot{\mathbf{z}}}(\mathbf{z}, \mathbf{p}, k)}{\partial \mathbf{z}}\right)^{\top} \mathbf{C}_{\mathbf{z}}\left(\frac{\partial \hat{\dot{\mathbf{z}}}(\mathbf{z}, \mathbf{p}, k)}{\partial \mathbf{z}}\right) .
$$

In order to justify the use of the loss function, it can be interpreted as a measure for the association probability of the two measurements. When defining an orbital state with a $\mathbf{p}$ - hypothesis using a fixed $k$, its probability given the two measurements is assessed using Bayes' theorem

$$
P(\mathbf{p} \mid \dot{\mathbf{z}}, \mathbf{z})=\frac{P(\dot{\mathbf{z}} \mid \mathbf{p}, \mathbf{z}) P(\mathbf{p} \mid \mathbf{z})}{P(\dot{\mathbf{z}} \mid \mathbf{z})},
$$

where the likelihood is given by

$$
P(\dot{\mathbf{z}} \mid \mathbf{p}, \mathbf{z})=\mathcal{N}\left(\dot{\mathbf{z}} ; \hat{\dot{\mathbf{z}}}(\mathbf{p}), \mathbf{C}_{\hat{\mathbf{z}}}(\mathbf{p})+\mathbf{C}_{\dot{\mathbf{z}}}\right)
$$

assuming normal probability density functions. As the evidence is independent of the free parameters and the prior is constant for all possible orbits, only the likelihood is assessed for the association probability. The hypothesis with the largest likelihood is accordingly the best fitting orbit. Alternatively, the free parameters can be found that minimize the exponent of the likelihood, i.e. loss function shown above. This comes with the advantage that 
the result is distributed according to the $\chi^{2}$-distribution and can be gated using predefined thresholds. Thus, if $\min \{L(\mathbf{p}, k)\}$ falls below the previously defined threshold, the hypothesis is accepted and both tracklets are associated to each other.

The topography of the loss function is again evaluated to study the suitability for computational optimization schemes. Fig. 7 shows the loss function for the same example case as for the initial-value method. Three individual discrete cases of the loss function defined by the allowed $k$ interval have been created. In contrast to the

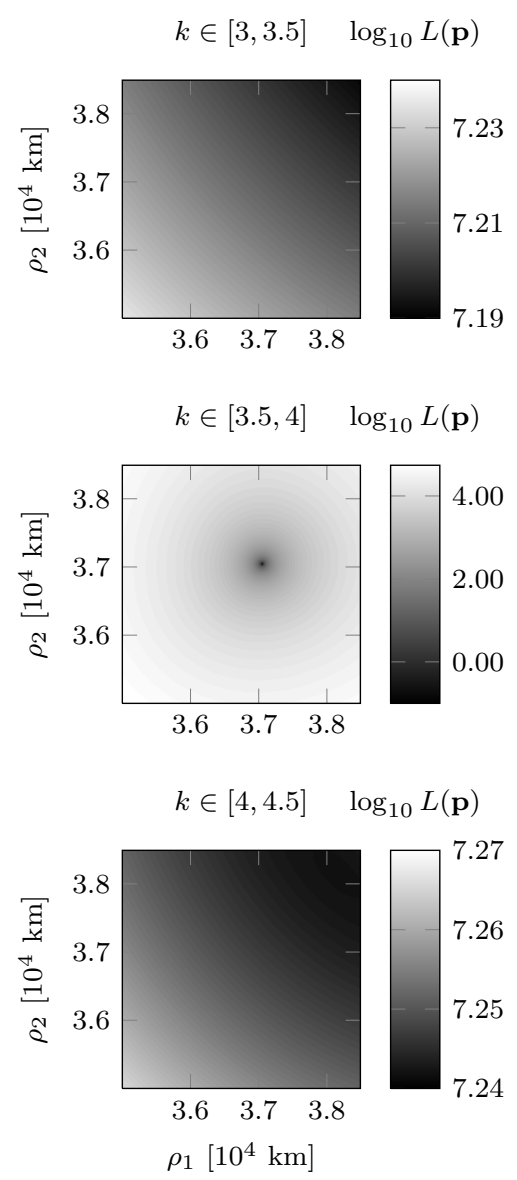

Figure 7: Loss function for boundary-value method. The same observations were used as in the previous figures 3 and 4 . The loss function is evaluated for different ranges of allowed orbital periods $k$.

initial-value method, each discrete case of the loss function contains only one local and therefore also global minimum. The global minimum for the overall problem is then ob- tained by identifying the value of $k$ that yields the smallest loss-function minimum.

\subsection{Admissible region}

As in the case of the initial-value formulation, the solution space for $\mathbf{p}$ - hypotheses is constrained to an admissible region $\mathcal{C}$. In principle, the complete information of the tracklets, i.e. line-of-sight and derivative, can be used to determine the extend of the feasible region. A methodology to obtain such an admissible region $\mathcal{C}(\mathbf{z}, \dot{\mathbf{z}})$ has been derived by Roscoe et al. (2013).

Here, the line-of-sight information alone is used to geometrically define a feasible range interval in order to simplify the process. Thus, the resulting region will have a rectangular shape. This is achieved by defining a smallest possible perigee $\left\|\mathbf{r}_{\min }\right\|$, a largest possible apogee radius $\left\|\mathbf{r}_{\max }\right\|$, and an allowed semi-major axis interval $\left[a_{\min }, a_{\max }\right]$ for all observations

$$
\begin{array}{r}
\mathcal{C}(\mathbf{z})=\left\{\rho_{i}, i \in[1,2]:\left\|\mathbf{r}_{\min }\right\|\right. \\
\leq\left\|\mathbf{r}\left(\rho_{i}\right)\right\| \leq\left\|\mathbf{r}_{\max }\right\|, \\
\left.a_{\min } \leq a\left(\rho_{1}, \rho_{2}\right) \leq a_{\max }\right\} .
\end{array}
$$

Following the derivations in (Schumacher et al., 2013; Roscoe et al., 2013) the range bounds

$$
\left\|\mathbf{r}_{\min }\right\|=a_{\min }\left(1-e_{\max }\right)
$$

and

$$
\left\|\mathbf{r}_{\max }\right\|=a_{\max }\left(1+e_{\max }\right)
$$

are computed given the semi-major axis interval and the greatest allowed eccentricity $e_{\max }$.

As illustrated in Fig. 8, the radius for a given range $\rho_{i}$ is given by

$$
\left\|\mathbf{r}_{i}\right\|^{2}=\rho_{i}^{2}+\left\|\mathbf{r}_{s, i}\right\|^{2}-2 \rho_{i} c_{i}
$$

where

$$
c_{i}=\left(\mathbf{r}_{s, i} \cdot \mathbf{u}_{i}\right)
$$

and $i$ is either 1 or 2 for the first or second observation respectively. The bounding range interval for each tracklet 


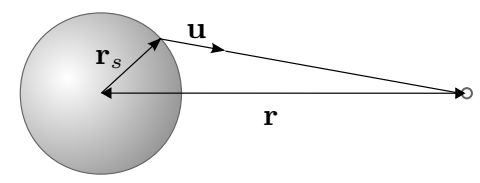

Figure 8: Illustration of the measurement geometry.

is then computed with the following equations, where (19) has been rearranged for the range.

$$
\begin{gathered}
\rho_{\min , i}=-c_{i}+\sqrt{c_{i}^{2}+\left\|\mathbf{r}_{\min }\right\|^{2}-\left\|\mathbf{r}_{s, i}\right\|^{2}} \\
\rho_{\max , i}=-c_{i}+\sqrt{c_{i}^{2}+\left\|\mathbf{r}_{\max }\right\|^{2}-\left\|\mathbf{r}_{s, i}\right\|^{2}}
\end{gathered}
$$

Additional to the range interval, the solutions to the Lambert's problem are also kept inside the semi-major axis bounds by defining an allowed interval of orbital revolutions

$$
\begin{aligned}
& k_{\min }=\left\lfloor 2 \Delta t / P\left(a_{\min }\right)\right\rfloor / 2 \\
& k_{\max }=\left\lfloor 2 \Delta t / P\left(a_{\max }\right)\right\rfloor / 2,
\end{aligned}
$$

using the orbital period $P$ and the time interval between the two tracklets $\Delta t=t_{2}-t_{1}$. The period is computed from the semi-major axis using Kepler's third law. The Lambert solver requires the number of completed full and half revolutions. Therefore, the values are rounded to the next lower 0.5 fraction.

\subsection{Computational optimization}

As mentioned above, the loss function $L(\mathbf{p}, k)$ must be minimized for all $k \in K=\left\{k_{\min }, k_{\min }+0.5, \ldots, k_{\max }\right\}$

$$
\mathbf{p}_{k, \min }=\underset{\mathbf{p} \in \mathcal{C}}{\arg \min } L(\mathbf{p}, k)
$$

As the loss function is smooth enough, optimization algorithms can use the gradient information to efficiently reach the minimum. Prominent examples are conjugate gradient or quasi-Newton methods. The latter has been used in this work and will be explained in the following.

The quasi-Newton methods approximate the loss function locally around some initial point $\mathbf{p}^{*}=\left(\rho_{1}^{*}, \rho_{2}^{*}\right)$ with a quadratic function. Then, the minimum is iteratively searched by finding the root of the gradient using Newton's method. If no analytical expression for the gradient is known, it can be provided using a finite difference scheme. Additionally, the inverse of the Hessian matrix of the loss function is required. Instead of computing it directly, it is approximated and iteratively improved during the minimization process. Different variants of the quasi-Newton method exists, resulting in different schemes on how to improve the matrix. An implementation for the popular Broyden-Fletcher-Goldfarb-Shanno (BGFS) scheme is used here and can be obtained from Press et al. (2007). For a detailed derivation of the algorithm refer to the book of Fletcher (1987).

As written above, the initial values $\mathbf{p}^{*}$ for the free parameters must be provided for each search iteration. That is why Kepler's third law is again used to determine a semi-major axis value $a(k)$ for each $k$ using the time interval between the tracklets. The initial value for each range is then computed by

$$
\rho_{i}^{*}=-c_{i}+\sqrt{c_{i}^{2}+a(k)^{2}-\left\|\mathbf{r}_{s, i}\right\|^{2}},
$$

assuming a circular orbit. This initial value is very close to the solution if the assumption is accurate. However, different schemes to define an initial value can be developed for other orbit types.

After evaluating all $k$ values, each one is selected that yields a loss function minimum below the predefined threshold. If at least one is obtained, the measurements are associated to each other with one orbit candidate. Multiple candidates are created if more $k$ values fulfill the condition. These must be then validated with further measurements.

\section{Assessment and comparison}

The proposed boundary-value method is now assessed in terms of association and run-time performance. For that purpose, the Astronomical Institute of the University of Bern (AIUB) provided a set of measurements of 
the ZimSMART telescope (Zimmerwald SMall Aperture Robotic Telescope). The telescope scans the sky for objects in near-geostationary orbits. The scan is achieved in an automatic way, where declination stripes for a fixed right ascension value are captured. The declination range is beforehand determined considering the space object density of the region. A typical tracklet contains three to five single observations and its duration is between one and two minutes. Therefore, the spacing between individual measurements of the tracklet is around 20 seconds. The characteristics of the measurement set are summarized in table 2 .

Herzog et al. (2013) assess the accuracy of the ZimSMART astronomic positions using precise positions of navigation satellites. In addition to the noise (around $0.7 "$ ) they observe a systematic offset caused by systematic errors in the recording of the exposure epoch. In case of the navigation satellites, along-track errors up to $10 "$ are obtained. That is why the uncertainty attached to the measurements is described with two different components, i.e. the accuracy and the precision (c.f. Siminski et al. (2013a)). The latter must be used to determine the standard deviation of angular rates, while the accuracy only influences the standard deviation of the angles.

The set was earlier processed by AIUB, i.e. the tracklets are tested with already cataloged objects. 180 tracklets could be successfully associated to previously observed objects. 40 of these objects have been observed two times in the measurement series, while 100 objects are only observed once. Although more tracklets are available, only the 180 tracklets that have been successfully associated are used for the performance test.

\subsection{Association performance}

Having no prior information, i.e. in the build-up phase of a catalog or when a set consists of only uncorrelated tracklets, each tracklet must be tested against all others. In an optimal test, all tracklets that belong to the same
Table 2: Specifications of the used measurements

\begin{tabular}{ll}
\hline Specification & Value \\
\hline First frame & Aug 18, 2012 22:59:08.64 UTC \\
Last frame & Aug 20, 2012 02:01:32.69 UTC \\
\hline Total no. tracklets & 204 \\
No. identified tracklets & 180 \\
No. detected objects & 140 \\
No. re-observed objects & 40 \\
\hline Tracklet duration & $1-2$ minutes \\
No. of measurements & $3-5$ per tracklet
\end{tabular}

object are associated to each other, while tracklets that do not are not grouped together.

The proposed optimization method for the boundaryvalue formulation is compared to the optimization method using the initial-value formulation. The association performance is tested by comparing the number of erroneous decisions for observations of non-common objects with the number of erroneous decisions for observations of common objects. The first is usually referred to as the false positive rate, while the latter describes the false negative associations. The all-vs-all tests are performed using different noise models in order to compare the robustness of the methods. The standard deviation of the noise is altered,i.e. $\sigma_{\alpha, \delta} \in\{0.7 ", 1.2 ", 2 "\}$. This uncertainty is used to calculate the deviation of the angular rates from a least squares fit. The systematic error is assumed to be distributed with a standard deviation of $5 \%$.

The results of the test are shown in Fig. 9, where min $L$ is computed for all tracklet pairs using the two formulations. The gating threshold is then altered to illustrate its effect on the respective association rates.

Both methods show similar results for the assumption $\sigma_{\alpha, \delta}=$ $0.7 "$ in that most tracklets that belong to each other were successfully associated, while the false positive rate remains low. The initial-value formulation, however, behaves more sensitive to an increase in the noise value assumption than the boundary-value one. 

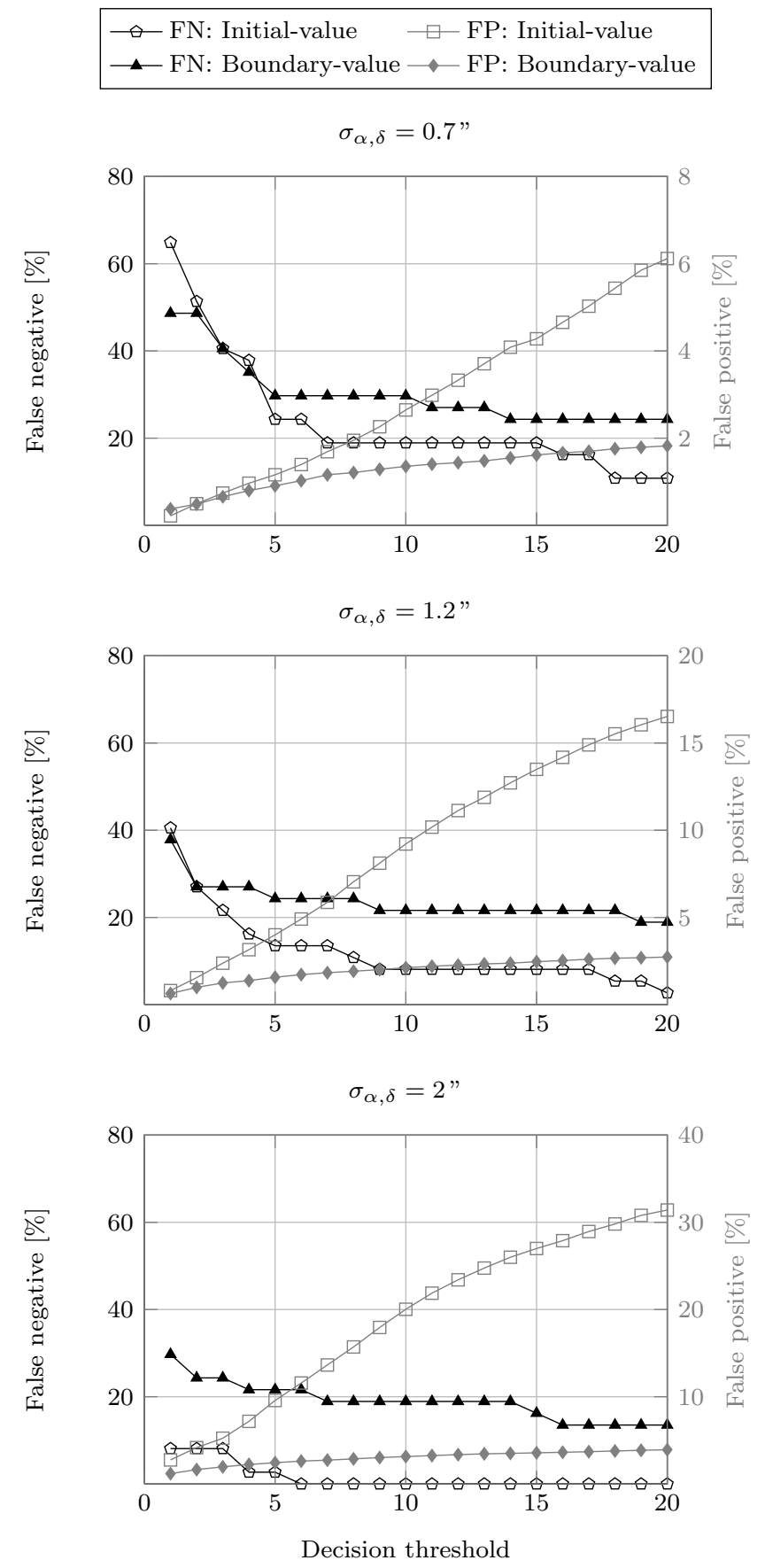

Figure 9: False positive (FP) and false negative (FN) ratio dependent on the maximum allowed minimum loss function value $\min L$ (Decision threshold) for different measurement noise assumptions $\sigma_{\alpha, \delta} \in\{0.7 ", 1.2 ", 2 "\}$.

In case of the initial-value formulation, a large uncertainty in the angular rates leads to even larger uncertainties in the modeled measurements. This ultimately leads to steeper ratio curves and more tracklet combinations being accepted as potential objects.
On the contrary, the impact of the different model is not that dominant when using the boundary-value formulation. The angular rates uncertainties scale $\min L$, which shifts the curves slightly but the general shape remains constant. Thus, if no accurate error model is available, the boundary-value method allows an easier calibration.

Each accepted candidate, i.e. each hypothesis which passes the decision threshold, needs to be taken into account for further measurement associations. A reasonable threshold must therefore be defined that keeps the false positive rate low but still accepts enough associations to fill the catalog. Further studies are necessary to define requirements for such a threshold.

\subsection{Run-time performance}

Another factor that needs to be assessed, especially when considering the large data flow of an optical sensor network, is the run-time performance. The all-vs-all test described above is used to estimate the computation time per tracklet pair. In total the number of association tests adds up to $\sim 16000$.

Both optimization approaches, one using the boundaryvalue and one the initial-value formulation, have been implemented in $\mathrm{C}++$ and are parallelized using OpenMP. The calculations for this work have been performed on an Intel Core i7-3720 CPU with $2.60 \mathrm{GHz}$ clock rate.

For the statistics shown in Fig. 9, the semi-major axis is forced to stay between 38000 and $45000 \mathrm{~km}$ to reduce the computational burden. The complete computation takes around 20 seconds for the boundary-value approach using a quasi-Newton method and 16 minutes for the initialvalue method. The latter uses a differential evolution minimization scheme with 15 search points per generation and a maximum number of 50 generations (c.f. with method by Storn and Price (1997)).

One way to compare the run-time performance independent from the used computer is to assess the number of loss function calls. The number of function calls differs 
from each tracklet pairing test to the other as the topography of the loss function can be more or less challenging for the optimization algorithm. That is why the worst case is estimated in the following.

With the given semi-major axis bounds, typically two discrete revolution cases need to be searched through for the provided data set using both methods. For a time separation of one day between the observations, the objects could have completed one complete or a half revolution. When considering a separation of two days, the semi-major axis bounds allows only 1.5 or 2 completed revolutions. Thus, the maximum total number of loss function calls for the initial-value method sums up to around 1500 , i.e. $2 \times 50$ generations $\times 15$ test points per generation. Similarly, the number of loss function calls for the boundary-value approach can be estimated. Typically each quasi-Newton search converges within 2-3 steps. A maximum number of steps has been defined to be 10 before the iteration is stopped. Therefore, even for the worst case, the total number of evaluations amount to 20 .

The run-time performance in terms of loss function calls can be compared with methods proposed by other researchers. Fujimoto et al. (2013) analyzes a similar data set and uses $5.8928 \times 10^{19}$ hypothesis bins for each pairing test to reach practical accuracy. Consequently, for each constellation this number of hypotheses must be propagated to the common epoch, i.e. the epoch of one observation. Thereafter, each hypotheses is compared with the bins of the other observation arc. Each propagation and the corresponding comparisons can be considered as approximately equally computationally demanding as one loss function call in the presented work in order to compare the different methods. DeMars and Jah (2013) choose a course grid to initialize their Gaussian mixture filter. For a simulated geostationary object, they sample the admissible region with 1000 points. All components of the filter are then individually propagated and compared to the next observation, which leads to a computational burden similar to 1000 function evaluations.

\subsection{Discussion}

The findings of the previous section are discussed in the following. Advantages and difficulties are compared for the boundary-value and initial-value formulation.

The initial-value method can incorporate any kind of orbit propagator and is not restricted to a purely Keplerian orbit model. It is well suited for use with numerical trajectory integration and enables use of fidelity force models. This is of particular interest for high-area-to-mass ratio (HAMR) objects affected by large non-gravitational perturbations. However, this would also add the solar radiation pressure coefficient as a free parameter to the optimization problem.

A major drawback is that the method is very sensitive to false noise model assumptions as it can be observed in section 5.1. Furthermore, as the loss function is dependent on both angles and angular rates, a false noise model might lead to a different shape that favors either angles or the rates. Then, in addition to a bad association performance, the obtained orbit solution might be also largely off the target.

The loss function for the initial-value formulation so far does not allow focusing on one valley at a time, i.e. the individual local minimums can not be separated easily. As an effect it can not be guaranteed to find the global minimum. Additionally, the computation time is increased as the admissible region must be sampled with a large number of initial start points for each search (c.f. with results in section 5.2).

The boundary-value formulation is a promising approach: the simple shape of the loss function enables the use of gradient based optimization methods which speeds up the computation. Furthermore, as the different local solutions are already separated by the Lambert solver itself, it is generally more stable than the initial-value method as the 
global minimum is definitely found. The separation also speeds up the computation as only one start point for each search iteration is used. The total number of loss function calls outperforms all other presented methods.

The used loss function relies solely on the angular rates. That makes it more tolerant against a false noise model as illustrated in section 5.1.

Traditional solutions to the Lambert's problem typically only account for two-body dynamics. This can cause difficulties when associating measurements that are separated by long time spans or objects with dominant nonconservative forces. However, this difficulty can be circumvented by using numerical orbit models and modern shooting methods for the Lambert's problem that also account for perturbations.

\section{Summary and Conclusion}

An advanced method for the association of tracklet pairs has been presented. The approach solves the tracklet association and initial orbit determination using a boundaryvalue formulation. It finds the best matching range pair given two tracklets at different observation epochs. It uses the solution to the Lambert's problem to calculate orbit candidates and the angular rates to distinguish between good and bad candidates. The initial orbit determination is reformulated into an optimization problem. The shape of the minimized loss function is very suitable for optimizers, which ultimately leads to a rapidly converging association and orbit determination process.

The approach promises to be a robust and fast replacement for current methods used in space object cataloging tasks. To confirm the suitability, an evaluation and comparison of the different approaches has been performed using a data set from the ZimSMART telescope. The results are summarized in the following. Firstly, current methods using an initial-value formulation are very computationally demanding. More importantly, they require very accurate knowledge on the errors and noise and are thus not very robust to false assumptions. Additionally, the loss function based on initial-value formulations is difficult to minimize as multiple local minimums must be identified. This study has shown that the presented approach can overcome this weaknesses. The optimization algorithm using the boundary-value formulation will definitely find the global minimum in the specified admissible region. Furthermore, the robustness w.r.t. to false noise model assumptions has been demonstrated. Lastly, the presented approach requires much less computational resources than other competing methods.

It is recommended that further research be undertaken in the assessment of noise and error models. These are required to define reasonable decision thresholds, which decide whether object candidates are created or not. Further investigations are also needed to determine which threshold and therefore false positive and false negative ratio is beneficial for an efficient but still all-encompassing catalog.

\section{References}

L. Ansalone and F. Curti. A genetic algorithm for Initial Orbit Determination from a too short arc optical observation. Advances in Space Research, 52:477-489, April 2013.

R. H. Battin. An Introduction to the Mathematics and Methods of Astrodynamics. AIAA, rev. ed. edition, 1999.

K.J. DeMars and M.K. Jah. Probabilistic Initial Orbit Determination Using Gaussian Mixture Models. Journal of Guidance, Control and Dynamics, 2013.

R. Fletcher. Practical methods of optimization. Wiley-Interscience, 1987. ISBN 0-471-91547-5.

C. Früh and T. Schildknecht. Catalogue Correlation of Space Debris Objects. In 5th European Conference on Space Debris, 2009.

K. Fujimoto, D.J. Scheeres, J. Herzog, and T. Schildknecht. Association of Short-Arc Optical Tracks via the Direct Bayesian Admissible Region: Theory and Application. In 6th European Conference on Space Debris, April 2013.

R. H. Gooding. A procedure for the solution of Lambert's orbital boundary-value problem. Celestial Mechanics and Dynamical Astronomy, 48(2):145-165, 1990.

J. Herzog, T. Schildknecht, A. Hinze, M. Ploner, and A. Vananti. Space Surveillance Observations at the AIUB Zimmerwald Ob- 
servatory. In 6th European Conference on Space Debris, April 2013.

A. Milani, G. Gronchi, M.D.M. Vitturi, and Z. Knezevic. Orbit Determination with Very Short Arcs. I Admissible Regions. Celestial Mechanics and Dynamical Astronomy, 90:57-85, 2004.

W.H. Press, S.A. Teukolsky, W.T. Vetterling, and B.P. Flannery. Numerical Recipes: The Art of Scientific Computing. Cambridge University Press, 3 edition, 2007.

C.W.T. Roscoe, P.W. Jr. Schumacher, and M.P. Wilkins. Parallel Track Initiation for Optical Space Surveillance Using range and Range-Rate Bounds. In AIAA/AAS Astrodynamics Specialist Conference, August 2013.

P.W. Jr. Schumacher, M.P. Wilkins, and C.W.T. Roscoe. Parallel algorithm for track initiation for optical space surveillance. In 6 th European Conference on Space Debris, 2013.

J. Siminski, H. Fiedler, and T. Schildknecht. Track Association Performance of the Best Hypotheses Search Method. In 6th European Conference on Space Debris, Darmstadt, Germany, April 2013a.

J. Siminski, O. Montenbruck, H. Fiedler, and M. Weigel. Best Hypotheses Search on Iso-Energy-Grid for Initial Orbit Determination and Track Association. In AAS/AIAA Spaceflight Mechanics Meeting, number 23rd, Kauai, Hawai, February 2013b.

Rainer Storn and Kenneth Price. Differential Evolution - A Simple and Efficient Heuristic for global Optimization over Continuous Spaces. Journal of Global Optimization, 11(4):341-359, 1997.

G. Tommei, A. Milani, and A. Rossi. Orbit Determination of Space Debris: Admissible Regions. Celestial Mechanics and Dynamical Astronomy, 97:289-304, 2007. 